\title{
Activation of RhoB in simvastatin-induced apoptosis of differentiated human skeletal muscle cells Timm Zörgiebel ${ }^{1}$, Lukas Weigl ${ }^{2}$, Harald Genth ${ }^{3}$ and Martin Hohenegger*1
}

\author{
Address: ${ }^{1}$ Institute of Pharmacology, Medical University of Vienna, Austria, ${ }^{2}$ Department of Anesthesiology and Critical Care Medicine, Medical \\ University of Vienna, Austria and ${ }^{3}$ Institute of Toxicology, Medical School Hannover, Germany \\ Email: Martin Hohenegger* - martin.hohenegger@meduniwien.ac.at \\ * Corresponding author
}

\author{
from I3th Scientific Symposium of the Austrian Pharmacological Society (APHAR). Joint Meeting with the Austrian Society of Toxicology (ASTOX) and the \\ Hungarian Society for Experimental and Clinical Pharmacology (MFT) \\ Vienna, Austria. 22-24 November 2007 \\ Published: 14 November 2007 \\ BMC Pharmacology 2007, 7(Suppl 2):A16 doi:10.1186/I47I-2210-7-S2-A16
}

This abstract is available from: http://www.biomedcentral.com/I47I-22I0/7/S2/AI6

(c) 2007 Zörgiebel et al; licensee BioMed Central Ltd.

Statins are well-tolerated cholesterol lowering drugs. However, in rare cases myotoxicity or even rhabdomyolysis occur. We have recently shown that in vitro statins are able to induce apoptosis in differentiated human skeletal muscle cells. In particular, simvastatin triggers the mitochondrial pathway of apoptosis via translocation of Bax from the cytosol to the mitochondrial membrane. Accordingly, caspase 9 and caspase 3 were consecutively activated. Here we show that an early event in simvastatin action is up-regulation of RhoB on mRNA level which is paralleled by the downstream Krüppel-like factor-2 (KLF2) and KLF-6. Moreover, the level of active, GTP-bound RhoB was increased in simvastatin-treated cells in a concentration-dependent manner as determined by Rhotekin pull-down assay. Up-regulation of RhoB on mRNA and protein level was dependent on HMG-CoA reductase inhibition. Interestingly, the up-regulation of RhoB was paralleled by caspase 2 activation, which might be directly associated with RhoB and therefore be required for the cytotoxic effect of simvastatin.

\section{Acknowledgements}

This work was supported by the GEN-AU Dragon Project to M.H. 